\title{
Evaluation Of Low-level Laser Therapy In Bilateral Tinnitus With Bilateral Sensorineural Hearing Loss
}

\section{Original Article}

\author{
Akram Masry, Ali K Mahrous and Ahmed Nabil El-Samnody \\ Department of Otorhinolaryngology, Faculty of Medicine, Al-Azhar University Hospitals, \\ Cairo, Egypt
}

\begin{abstract}
Background: Sound perception in the ear without external source is defined as tinnitus. In recent years, low level laser therapy has been widely used in the treatment of tinnitus.

Objective: To evaluate the effectiveness of low level laser therapy in the treatment of bilateral subjective tinnitus concomitant with bilateral sensorineural hearing loss.

Methods: A total of 30 patients with bilateral sensorineural hearing loss accompanied with bilateral subjective tinnitus collected from outpatient clinics of otorhinolaryngology department in Al-Azhar University hospitals and National Hearing and Speech Institute, during the period from September 2018 to December 2019. Tinnitus Handicap Inventory (THI) \& Visual Analogue Scales (VAS) were assessed. All the patients received Low-Level Laser Therapy in one ear and a placebo in the other ear. Follow-up period was for 3 and 6 months.

Results: As regards VAS score, the mean of the active laser group of patients was (8) before the sessions which changed to (5.1) 3 months after completion of the sessions, while it worsened to (7.5) 6 months after completion of the sessions. As regards THI severity score, the mean of tinnitus handicap inventory was (54.7) before the sessions and became (38.1) 3 months after the sessions but worsened 6 months after the sessions (49.1). There was non-statistically significant change in VAS and THI score from baseline to 6 months post-intervention.

Conclusion: Low-Level Laser Therapy for sensorineural tinnitus seemed to be a safe method in reducing the severity of tinnitus, but there was non-statistically significant difference from baseline to 6 months post-intervention.
\end{abstract}

Key Words: LASER, sensorineural hearing loss, subjective tinnitus.

Received: 12 March 2021, Accepted: 15 April 2021

Corresponding Author: Akram Masry, Department of Otorhinolaryngology, Faculty of Medicine, Al-Azhar University Hospitals, Cairo, Egypt, Tel.: +20 100234563, E-mail: akrammasry88@gmail.com

ISSN: 2090-0740, 2021

\section{INTRODUCTION}

Sound perception in the ear without external source is defined as tinnitus. About $20 \%$ of adults who experience tinnitus will require clinical intervention. Tinnitus itself is not a disease, but it is actually a symptom that can be associated with multiple diseases and affected by many factors $^{[1]}$.

Sensorineural tinnitus represents a high percentage of the cases of chronic tinnitus. Causes include presbyacusis, sudden sensorineural hearing loss, noise induced hearing loss, Meniere's disease (MD), Hearing loss induced by ototoxic drugs and cochlear otosclerosis ${ }^{[2]}$.

A variety of treatment models has been tried to reduce tinnitus severity, such as pharmacological treatment (e.g. psychoactive drugs, vasodilators and antioxidants), Tinnitus Retraining Therapy (TRT) and Cognitive Behavioral Therapy $(\mathrm{CBT})^{[3]}$.

In recent years, low level laser therapy has been widely used in the treatment of tinnitus. This treatment has previously been reported to be useful for wound healing, musculoskeletal diseases and pain control. Published studies give conflicting success rates, from 80 percent to no significant improvement in the relief of tinnitus ${ }^{[4]}$.

In this study, we aimed to investigate the effectiveness of low-laser laser therapy for tinnitus, using a new device permitting very low power laser output, on a short term treatment schedule.

\section{MATERIALS AND METHODS}

This randomized, placebo-controlled, double-blind study included 30 patients collected from outpatient clinics of otorhinolaryngology department in Al-Azhar University hospitals and National Hearing and Speech Institute, during the period from September 2018 to December 2019. The essential presentation of all the patients that they were complaining of bilateral sensorineural hearing loss accompanied with bilateral subjective tinnitus. Exclusion criteria of the study include patients with objective tinnitus, patients under 18 years, patients with conductive or mixed hearing loss with tinnitus, and patients with sensorineural hearing loss due to known cause 
(e.g. acoustic shwannoma). All the patients were subjected to thorough otorhinolaryngological examination, audiological assessment in the form of pure tone audiometry with tympanometry. Tinnitus Handicap Inventory (THI) \& Visual Analogue Scales (VAS) were collected for each patient according to his answers before and after receiving the laser sessions.

All the patients received Low-Level Laser Therapy in one ear and a placebo in the other ear (applying the laser device without activating it), in the form of twenty-one sessions, three sessions per week for seven weeks, fifteen minutes per session for each ear, using Tinnitool (Dismark $\mathrm{GmbH}$, Maur, Switzerland)with intensity of $5 \mathrm{~mW}$ and wavelength of $650 \mathrm{~nm}$. Both active laser and placebo procedures were administered in the same session, in a similar manner, starting with the active session. The LowLevel Laser system is composed of two parts, a low-power soft laser emitter (Medic Laser) and a fiberglass connector with a wearable head band directing the low-power laser through the external ear canal, and into the inner ear (Tinnitool). Medic Laser together with Tinnitool was able to produce low-power laser that was able to penetrate skin and mucosa up to approximately $1.8 \mathrm{~cm}$ depth. All the patients were followed up after finishing all the sessions for 3 and 6 months by direct meeting or phone contact.

All statistical analyses were performed at a 5\% confidence interval and a $p$-value $<0.05$ was considered significant. The statistical software packages SAS 9.2 (SAS Institute, Cary, NC, USA) and SPSS 15.0 (SPSS Inc., Chicago, IL, USA) were used for the analysis of the data. In addition to the standard descriptive statistical calculations as mean, standard deviation (SD) (min-max), the results on categorical measurements were presented in numbers (\%). The mean and SD of hearing loss in $\mathrm{dB}$ pre and post intervention were compared using the paired t-test.

\section{RESULTS}

The mean age of patients in our study was 36.3 years (18-55 years) with standard deviation (12.4). Male age ranged from 22 to 55 years with mean age (38.4), and female age ranged from 18 to 51 years with mean age (34.8). The patients were 16 male and 14 female with variable degrees of bilateral sensorineural hearing loss and bilateral subjective tinnitus. As regards sex, there were 14 females $(46.7 \%)$ and 16 males $(53.3 \%)$ in our study (Table1).

Table 1: Patient characteristics

\begin{tabular}{|c|c|c|}
\hline \multicolumn{2}{|c|}{ Patient data } & No. $(\%)$ \\
\hline \multicolumn{2}{|c|}{ Age } & Mean $\pm \mathrm{SD}=36.3 \pm 12.4$ \\
\hline \multirow{2}{*}{ Sex } & Male & $16(53.3 \%)$ \\
\hline & Female & $14(46.7 \%)$ \\
\hline
\end{tabular}

The mean of the VAS score was (8) before the sessions which changed to (5.5) 3 months after completion of the sessions that was statistically significant relief as regards pre-intervention VAS and 3 months post-intervention VAS $(8.0 \pm 2.0$ vs. $5.5 \pm 2.1)$. The mean of the VAS score was (8) before the sessions which changed to (7.5) 6 months after completion of the sessions that wasn't statistically significant difference as regards pre-intervention VAS and 6 months post-intervention VAS $(8.0 \pm 2.0$ vs. $7.5 \pm 1.5)$ (Table 2$)$.

Table 2: VAS score before and after sessions (3 and 6 months)

\begin{tabular}{cccc}
\hline VAS & Mean & SD & $P_{\text {-value }}^{\#}$ \\
\hline Before & 8 & 2 & $0.013^{*}$ \\
After 3 months & 5.5 & 2.1 & \\
Before & 8 & 2 & $0.142^{* *}$ \\
After 6 months & 7.5 & 1.5 & \\
\hline \#Paired-t test & *Significant & $* *$ Non-significant & \\
The mean of the THI score was $\quad(54.7)$ before
\end{tabular}

the sessions which changed to (38.1) 3 months after completion of the sessions that was statistically significant relief as regards pre-intervention THI and 3 months postintervention THI $(54.7 \pm 16.3$ vs. $38.1 \pm 16.4)$. The mean of the THI score was (54.7) before the sessions which changed to (49.1) 6 months after completion of the sessions that wasn't statistically significant difference as regards pre-intervention THI and 6 months post-intervention THI $(54.7 \pm 16.3 .0$ vs. $49.1 \pm 12.6)($ Table 3$)$.

Table 3: Tinnitus Handicap Severity score before and after sessions ( 3 and 6 months)

\begin{tabular}{cccc}
\hline VAS & Mean & SD & P-value $^{\#}$ \\
\hline Before & 54.7 & 16.3 & $<0.0001^{*}$ \\
After 3 months & 38.1 & 16.4 & \\
Before & 54.7 & 16.3 & $0.147^{* *}$ \\
After 6 months & 49.1 & 12.6 & \\
\hline
\end{tabular}

\#Paired-t test $\quad *$ Significant $\quad * *$ Non-significant

As regards grading of THI severity score, grade 1 of tinnitus was not identified in any patient before intervention but it was detected in 8 patients after 3 months and 2 patients after 6 months. Grade 2 was detected in 4 out of 30 before, in 14 out of 30 after 3 months and in 12 out of 30 after 6 months. As regards grade 3, it was recognized in 10 out of 30 patients at baseline which slightly decreased to 6 out of 303 months after intervention then increased to 12 out of 30 after 6 months. Prevalence of Grade 4 decreased significantly from 14 out of 30 at baseline to 4 out of 303 months after intervention then increased to 4 out of 30 after 6 month. Grade 5 of tinnitus was identified in 2 patients before intervention but it was not detected after 3 and 6 months (Table 4). 
Table 4: Tinnitus Handicap Severity score in grades before and after sessions

\begin{tabular}{|c|c|c|c|c|c|c|c|}
\hline \multirow{2}{*}{ Grades } & \multicolumn{2}{|c|}{ Before } & \multicolumn{2}{|c|}{ After 3 months } & \multicolumn{2}{|c|}{ After 6 months } & \multirow{2}{*}{$P$-value $e^{\#}$} \\
\hline & $\mathrm{N}$ & $\%$ & $\mathrm{~N}$ & $\%$ & $\mathrm{~N}$ & $\%$ & \\
\hline Grade 1 & 0 & $0.0 \%$ & 8 & $26.6 \%$ & 2 & $6.7 \%$ & \multirow{5}{*}{$\begin{array}{c}0.04 * \text { for } 3 \text { months follow-up } \\
0.406^{* *} \text { for } 6 \text { months follow-up }\end{array}$} \\
\hline Grade 2 & 4 & $13.3 \%$ & 14 & $46.7 \%$ & 12 & $40.0 \%$ & \\
\hline Grade 3 & 10 & $33.3 \%$ & 6 & $20 \%$ & 12 & $40.0 \%$ & \\
\hline Grade 4 & 14 & $46.7 \%$ & 2 & $6.7 \%$ & 4 & $13.3 \%$ & \\
\hline Grade 5 & 2 & $6.7 \%$ & 0 & $0.0 \%$ & 0 & $0.0 \%$ & \\
\hline
\end{tabular}

\#chi-square test *Significant $\quad * *$ Non-significant

\section{DISCUSSION}

Tinnitus is the perception of a "phantom" sound that does not actually exist. It often is a disabling symptom of different disorders affecting the auditory system, and it can be associated with a wide range of pathological conditions. Tinnitus must be distinguished from real auditory hallucination, which is a symptom of psychiatric or neurological disorders ${ }^{[5]}$.

Tinnitus management options that have been subjected to randomized controlled trial (RCT) investigation include pharmacological interventions, sound-based interventions, psychological interventions, magnetic stimulation, electrical stimulation, manual physical therapy, relaxation therapy, complementary and alternative medicine (CAM) therapies, education and information, self-help interventions and complex interventions (defined as a combination of two or more of the preceding modalities) ${ }^{[6]}$.

Treatment with Low-Level Laser Therapy has been tried in some studies as a promising treatment to reduce the severity and frequency of tinnitus. The mechanism of action of low power laser on the inner ear and on tinnitus is not well understood. With regard to the metabolism of cells, non-invasive, low power laser acts very positively by enhancing oxidative activity and protein synthesis. Another proposed mechanism is blood flow increase associated with suppression of the sympathetic nerve action potential. Low level laser has been suspected to improve local microcirculation and to increase oxygen supply to hypoxic cells $^{[7,8]}$.

The mean age of patients in our study was 36.3 years (18-55 years) with standard deviation (12.4). Male age ranged from 22 to 55 years with mean age (38.4), and female age ranged from 18 to 51 years with mean age (34.8). The patients were 16 male and 14 female with variable degrees of bilateral sensorineural hearing loss and bilateral subjective tinnitus. As regards sex, there were 14 females $(46.7 \%)$ and 16 males $(53.3 \%)$ in our study. In 2009, Teggi et al., conducted a study on 54 patients divided into 2 groups, 27 patients in active laser group (11 females and 16 males) with mean age 51.6, while the placebo group consisted of 27 patients (13 females and 14 males) with mean age 53.1.

The mean of the VAS score was (8) before the sessions which changed to (5.5) 3 months after completion of the sessions that was statistically significant relief as regards pre-intervention VAS and 3 months post-intervention VAS. The mean of the VAS score was (8) before the sessions which changed to (7.5) 6 months after completion of the sessions that wasn't statistically significant difference as regards pre-intervention VAS and 6 months postintervention VAS. The mean of the THI score was (54.7) before the sessions which changed to (38.1) 3 months after completion of the sessions that was statistically significant relief as regards pre-intervention THI and 3 months postintervention THI. The mean of the THI score was (54.7) before the sessions which changed to (49.1) 6 months after completion of the sessions that wasn't statistically significant difference as regards pre-intervention THI and 6 months post-intervention THI.

Teggi et al. studied patients divided into two groups, the first group received active laser therapy $20 \mathrm{~min}$ a day for 3 months with a $650-\mathrm{nm}, 5-\mathrm{mW}$ soft laser device, and the second group used a dummy device which duplicated all aspects of active laser therapy except for the activation of the laser beam. They used THI and VAS to assess the response of patients to LLLT and placebo. They showed that THI total score was decreased in both groups as well as in our results, this study showed no statistical difference between the two groups. Visual analogue scale (VAS) for the intensity of tinnitus did not change in either group. This study failed to demonstrate the efficacy of LLLT as a single therapy for tinnitus ${ }^{[9]}$.

In a double-blind randomized study, Mirz et al. (1999) found only moderate (18\%) subjective improvement in measurements of tinnitus loudness, visual analog scale (VAS) scores, and Tinnitus Handicap Inventory (THI) scores with no statistically significant differences between the effects of active laser and placebo treatments. They concluded that no significant therapeutic effects were found $d^{[10]}$.

Rogowski et al.(1999) reported no significant difference in annoyance and loudness of tinnitus or changes in transient evoked otoacoustic emission amplitude between laser and placebo in a double-blind randomized study; while in our study, we found decrease in annoyance and loudness (measured by THI and VAS) in both groups, with higher percentage of reduction in the active laser group ${ }^{[1]}$. 
In a study on 120 patients with chronic tinnitus, Hahn et al. (2001) reported an improvement in tinnitus and pure tone audiometry after 10 sessions of laser therapy, each lasting for $10 \mathrm{~min}$; laser therapy was combined with Ginkgo biloba, unlike our study in which the session lasted for 15 minutes, 21 sessions with no combination with other treatment method. A continuous beam was applied initially, followed by a pulse beam, each for $5 \mathrm{~min}$, using a $830 \mathrm{~nm} / 200 \mathrm{mV}$ laser probe. Tinnitus disappeared in one case and worsened in one case. Improvement in tinnitus was confirmed in 61 cases $(50.8 \%)$ and tinnitus remained unchanged in the other 57 cases $(47.5 \%)^{[12]}$.

Nakashima et al. (2002) observed no significant differences between patients performing active or placebo laser therapy with regard to loudness, duration, quality, and annoyance of tinnitus. The study included 68 ears in 45 patients (16 men and 29 women) with disabling unilateral or bilateral tinnitus with SNHL. In the active laser, the output was $60 \mathrm{~mW}$, and the wavelength was $810 \mathrm{~nm}$. Patients' mean age was $53.7 \pm 12.8$ years. The active or placebo laser treatment was administered transmeatally once a week for 6 minutes. Laser irradiation was performed four times during a 4 -week period ${ }^{[13]}$.

In 2012, Salahaldin et al. studied 101 ears of 65 patients aged 15-76 years with chronic unilateral or bilateral tinnitus ( 36 bilateral and 29 unilateral). A $5 \mathrm{~mW}$ laser with a wavelength of $650 \mathrm{~nm}$ (using Tinnitool ${ }^{\mathrm{TM}}$ ) was applied transmeatally for 20 minutes once daily for 3 months. Over half of the patients $(56.9 \%)$ had some form of improvement in their tinnitus symptoms. Mild improvement was reported in $33.8 \%$ of patients, moderate improvement was reported in $16.9 \%$, and full improvement was reported in $6.15 \%$. Of the patients who reported dizzy spells as a symptom of their tinnitus condition, $27.7 \%$ reported mild improvement and $16.9 \%$ reported full improvement ${ }^{[14]}$.

Also Mirvakili et al. 2014 performed a cross-sectional study on 120 patients with sensorineural hearing loss referred to their clinic between 2011 and 2012. Those older than 18 years-old with tinnitus for more than one year due to sensorineural hearing loss entered the study. Those with conductive or mixed hearing loss, and duration of less than one year were excluded. Before the study, at the end of treatment period and 3 months after the study, tinnitus severity was evaluated by visual analog scaling and tinnitus handicap inventory. The patients were randomly divided into two groups, 60 patients were placed in treatment group and another 60 patients were placed in the control group without any treatment. A low-level laser device (TINNImed, made in Switzerland) with intensity of $5 \mathrm{~mW}$ and wavelength of $650 \mathrm{~nm}$ was used.THI grade mean was $(2.73 \pm 0.79$ vs. $3.01 \pm 1.12)$ in control and therapy group before beginning the study and there was not any significant statistical difference between both groups $(\mathrm{P}=0.11)$. At the end of study, significant statistical difference was seen between the two groups $(\mathrm{P}=0.01)$ and three months after the end of therapy, the difference between both groups was not significant $(\mathrm{P}=0.85)^{[15]}$.
Mirvakili et al. 2014 showed that the obtained results from VAS criterion were investigated and measured in three time sections and then were compared between both groups. The obtained results from comparing the THI and VAS criteria among the patients of both groups indicate that radiation of low level laser can lead to recovery from the patients' tinnitus situation, particularly for short time, although, following the patients' tinnitus situation after three months from end of therapy propounds this hypothesis that its effects decreases with passing time ${ }^{[15]}$.

\section{CONCLUSION}

Low-Level Laser Therapy for sensorineural tinnitus seemed to be a safe method in reducing the severity of tinnitus, but in prolonged follow-up to the patients, there was non-statistically significant difference from baseline to 6 months post-intervention. However, the potential therapeutic effects of low power laser on tinnitus are still obscure. Although our results are encouraging, further studies are required in order to optimize the laser treatment regimen and to exclude side effects.

\section{CONFLICT OF INTERESTS}

There are no Conflicts of interest.

\section{REFERENCES}

1. Tunkel DE, Bauer CA, Sun GH, Rosenfeld RM, Chandrasekhar SS, Eugene R, et al. Head and Neck Surgery Clinical Practice Guideline: Tinnitus. 2014; 12(2)234-7.

2. Zeman F, Koller M, Figueiredo R, Aazevedo A, Rates $\mathrm{M}$, Coelho $\mathrm{C}$, et al. Tinnitus handicap inventory for evaluating treatment effects: which changes are clinically relevant? Otolaryngology Head and Neck Surgery. 2011; 145:282-287.

3. Shargorodsky J, Curhan GC, Farwell WR. Prevalence and characteristics of tinnitus among US adults. Am J Med. 2010; 123:711-718.

4. Tauber S, Beyer W, Schorn K, Baumgartner R. Transmeatal cochlear laser (TCL) treatment of cochlear dysfunction: A feasibility study for chronic tinnitus. Lasers in Medical Science. 2003; 18(3):154-161.

5. Baguley D, Mcferran D, Hall D. Tinnitus. Lancet. 2013; (22)382.

6. McFerran DJ, Stockdale D, Holme R, Large $\mathrm{CH}$, Baguley DM. Why is there no cure for tinnitus? Frontiers in Neuroscience. 2019; 13(5):1-13.

7. Ngao CF, Tan TS, Narayanan P, Raman R. The effectiveness of transmeatal low-power laser stimulation in treating tinnitus. European Archives of Oto-Rhino-Laryngology. 2014; 271(5):975-980.

8. Gungor A, Dogru S, Cincik H. Effectiveness of transmeatal low power laser irradiation for chronic tinnitus. J LaryngolOtol.2007; 12:1-5. 
9. Teggi R, Bellini C, Piccioni LO, Palonta F, Bussi M. Transmeatal low-level laser therapy for chronic tinnitus with cochlear dysfunction. Audiology and Neurootology. 2009; 14(2):115-120.

10. Mirz F, Zachariae R, Andersen SE, Nielsen AG, Johansen LV, Bjerring $\mathrm{P}$, et al. The low-power laser in the treatment of tinnitus. Clinical Otolaryngology and Allied Sciences. 1999; 24(4):346-354.

11. Rogowski M, Mnich S, Gindzieńska E, Lazarczyk B. Laser vs placebo [Low-power laser in the treatment of tinnitus--a placebo-controlled study]. OtolaryngolPol. 1999; 53(3):315-20.

12. Hahn A, Sejna I, Stolbova K, Cocek A. Combined laser-EGb 761 tinnitus therapy. Acta Otolaryngol Suppl. 2001; 545:92-3.
13. Nakashima $T$, Ueda $H$, Misawa $H$, Suzuki $T$, Tominaga $\mathrm{M}$, Ito $\mathrm{A}$, et al. Transmeatal low-power laser irradiation for tinnitus. Otology and Neurotology 2002; 23(3):296-300.

14. Salahaldin AH, Abdulhadi K, Najjar N, Bener A. LowLevel Laser Therapy in Patients with Complaints of Tinnitus: A Clinical Study. ISRN Otolaryngology. 2012; 1-5.

15. Mirvakili A., Mehrparvar A., Mostaghaci M., Mollasadeghi A., Mirvakili M.,Baradaranfar M, et al. Low level laser effect in treatment of patients with intractable tinnitus due to sensorineural hearing loss. Journal of Lasers in Medical Sciences. 2014; 5(2), 71-74. 